\title{
Style-Aware Image Recommendation for Social Media Marketing
}

\author{
Yiwei Zhang \\ The University of Tokyo \\ Tokyo, Japan \\ zhangyiwei@cvm.t.u-tokyo.ac.jp
}

\begin{abstract}
Social media have become a popular platform for brands to allocate marketing budget and build their relationship with customers. Posting images with a consistent concept on social media helps customers recognize, remember, and consider brands. This strategy is known as brand concept consistency in marketing literature. Consequently, brands spend immense manpower and financial resources in choosing which images to post or repost. Therefore, automatically recommending images with a consistent brand concept is a necessary task for social media marketing. In this paper, we propose a content-based recommendation system that learns the concept of brands and recommends images that are coherent with the brand. Specifically, brand representation is performed from the brand posts on social media. Existing methods rely on visual features extracted by pre-trained neural networks, which can represent objects in the image but not the style of the image. To bridge this gap, a framework using both object and style vectors as input is proposed to learn the brand representation. In addition, we show that the proposed method can not only be applied to brands but also be applied to influencers. We collected a new Instagram influencer dataset, consisting of 616 influencers and about 1 million images, which can greatly benefit future research in this area. The experimental results on two large-scale Instagram datasets show the superiority of the proposed method over state-of-the-art methods.
\end{abstract}

\section{CCS CONCEPTS}

- Information systems $\rightarrow$ Social recommendation; Content ranking; Social advertising.

\section{KEYWORDS}

Image Recommendation, Image Style, Content Discovery, Social Media Marketing

\section{ACM Reference Format:}

Yiwei Zhang and Toshihiko Yamasaki. 2021. Style-Aware Image Recommendation for Social Media Marketing. In Proceedings of the 29th ACM International Conference on Multimedia (MM '21), October 20-24, 2021, Virtual Event, China. ACM, New York, NY, USA, 9 pages. https://doi.org/10. $1145 / 3474085.3475453$

Permission to make digital or hard copies of all or part of this work for personal or classroom use is granted without fee provided that copies are not made or distributed for profit or commercial advantage and that copies bear this notice and the full citation on the first page. Copyrights for components of this work owned by others than ACM must be honored. Abstracting with credit is permitted. To copy otherwise, or republish, to post on servers or to redistribute to lists, requires prior specific permission and/or a fee. Request permissions from permissions@acm.org.

MM '21, October 20-24, 2021, Virtual Event, China

(c) 2021 Association for Computing Machinery.

ACM ISBN 978-1-4503-8651-7/21/10 ..\$15.00

https://doi.org/10.1145/3474085.3475453

\author{
Toshihiko Yamasaki \\ The University of Tokyo \\ Tokyo, Japan \\ yamasaki@cvm.t.u-tokyo.ac.jp
}

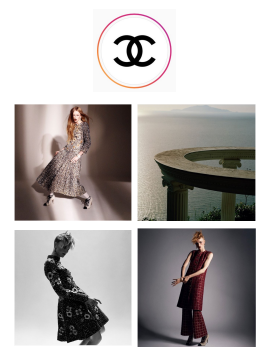

Images posted by the brand (a) chanelofficial in the past
Brand: need to select new images to post

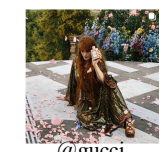
(a) gucci

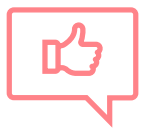

These images match the brand concept

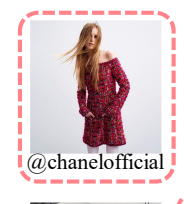

@ chanelofficial
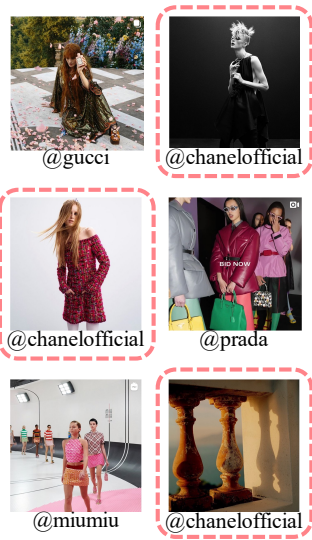

Figure 1: Illustration of recommending images based on brand's past posts on social media. Sample posts are original posted by the Instagram accounts shown below the images.

\section{INTRODUCTION}

Social media are a trending platform for brand marketing. A study reported that $90 \%$ of people buy from brands they follow on social media ${ }^{1}$. Researchers have also shown that establishing brand communities on social media has positive effects on brand loyalty $[25,26]$. Therefore, cultivating relationships with customers on social media has become an important part of brand marketing strategy and is primarily accomplished by posting images. Brands usually hire professional marketing groups to create and post images on image-sharing platforms, such as Instagram ${ }^{2}$. Meanwhile, brands might hire influencers to post images and help them promote products, because a research has shown that customers are more likely to trust advertisements by influencers rather than conventional marketing strategies [40]. In addition, brands often interact with customers by reposting their images. Discovering such user generated content help brands strengthen their relationships with customers [8]. However, such works have been performed only by experts so far, resulting in the investment of immense workforce and financial resources for selecting which images to post and repost. For famous brands, they may be able to hire professional groups to help them create and select the best image. For small brands or individual influencers, on the other hand, they may not be able to afford such expenses. For these reasons, we believe that

\footnotetext{
${ }^{1}$ https://www.marketingdive.com/news/90-of-people-buy-from-brands-theyfollow-on-social-media-study-says/577882/

${ }^{2}$ https://www.instagram.com
} 
automatically recommending images to brands is a necessary task and could be a powerful marketing tool.

Image recommendation to brands is hard to implement because identifying suitable images is difficult. Brands would post images that are not only related to the brand products but also represent the brand concept or lifestyle on social media. For instance, the fashion brand "Chanel" often posts images of products, such as clothes and bags, as well as landscape-related images. Therefore, only identifying objects in the image is not enough to define a brand To address this problem, we have investigated the homepages of many brands on Instagram. We found that, although a brand may post images with various objects, most images usually have a uniform image style. We observed that many famous brands maintain such concept consistency across social media channels. This strategy, called brand concept consistency in marketing literature, assists consumers to categorize brands in terms of their attributes, beliefs, and product-evoked emotions [24]. Studies have shown that brand consistency is the key to successful branding $[28,30]$. Therefore, we believe that the identification of the general style of a brand is more important than object recognition in their posted images. For influencers, we found that they show similar tendency when posting images on social media. We think image recommendation to brands and influencers are the similar task. Hence, a general model for style-aware image recommendation is needed. Figure 1 illustrates the proposed method of image recommendation based on the brand concept.

Recently, deep neural networks have been successfully deployed for tasks such as image recognition and object detection. However, learning the style of an image remains challenging. Moreover, the lifestyle or concept of brands is difficult to determine. In this work, we propose a method of learning brand concept representation and recommending images that match the brand concept. We assumed that images posted by the brand in the past reflect the brand concept. We considered both the objects and style of the image to be important in defining the brand concept. Regarding the objects, we used features extracted from the first fully connected (FC) layer with pre-trained convolutional neural networks $(\mathrm{CNN})$ as the object vector. Regarding the image style, we investigated the usefulness of correlations between feature maps of CNNs. We reduced the Gram matrix [14] of feature maps by incremental principal component analysis (PCA) [37] and used it as the style vector. Then, we trained a pairwise learning model to learn the brand concept representation. We evaluated our model using two large-scale Instagram datasets including a brand dataset and an influencer dataset. The experimental results show that the proposed method exhibits the best performance.

To summarize, our main contributions are as follows:

- We present an approach that integrates both object and style features for learning brand representation and recommending images that match the brand concept.

- The proposed framework can be applied to other tasks such as influencer marketing by only changing the input. In this paper, we collected an Instagram influencer dataset, including 616 influencers' information and about 1 million images. We release the Instagram influencer dataset to the public ${ }^{3}$.

$\overline{{ }^{3} \text { https://github.com/yiwei51/mm21_influencer_dataset }}$
- We conducted comprehensive experiments on two largescale Instagram datasets to demonstrate the effectiveness of our method.

\section{RELATED WORKS}

\subsection{Social Media Marketing}

Social media marketing is the use of social media platforms to promote products or services [10]. Brands are increasingly realizing the value of social media as they help brands easily reach their customers. Such trends also provide researchers with a new opportunity to analyze rich data generated via social media. Researchers have studied brands in various topics. Several works have analyzed the social media posts of brands to determine the features that positively impact brand popularity [5, 31]. Zhang et al. measured the relationship between brands to help them find potential business partners [43]. Kim et al. used clustering-based methods to detect visual brand associations from social images [21].

Nowadays, influencer marketing becomes an essential part of social media marketing strategies, as brands often hire influencers to promote products or services. Farseev et al. showed a demonstration of utilizing social multimedia for influencer discovery [9]. Gan et al. used a multi-modal network to recommend influencers to brands [11]. Kim et al. designed a model to classify influencers into specific categories [22].

\subsection{Image Style}

Early works related to image style analysis focus on image characteristics, such as color, line, composition, and intensity. Palermo et al. used a color histogram to determine the date of historical color images [35]. Lowe et al. proved that scale-invariant feature transform descriptors are effective for image style analysis because of their robustness to complex deformation and illumination changes [29]. Oliva et al. proposed a low-dimensional representation of the scenes called GIST descriptors, which can extract the spatial structure of an image and effective in scene classification [34].

Recently, deep feature representations have become increasingly popular in image style analysis. Karayev et al. used features extracted from a deep convolutional network to represent an image and further used it in a style classification task [20]. Gatys et al. found that correlations between feature maps of a CNN can be a good representation of image style [13]. Further, texture information is considered to be closely related to the style of an image [12]. In [27], they found that merging the intermediate feature maps demonstrated good performance in image inpainting task. In research on neural style transfer, a novel style representation, called the Gram matrix, has been proposed and deployed for transforming an image into a specified painting style while preserving its contents [14]. Several researchers have verified the effectiveness of the Gram matrix in oil painting image-classification tasks $[2,4]$.

\subsection{Image-Based Recommendation}

Image data are especially useful in cold-start scenarios of recommendation tasks and can also alleviate the sparsity problem. Therefore, in this research field, images are usually used as auxiliary information. In the pairwise learning framework called Bayesian personalized ranking (BPR), positive items are ranked higher than negative 


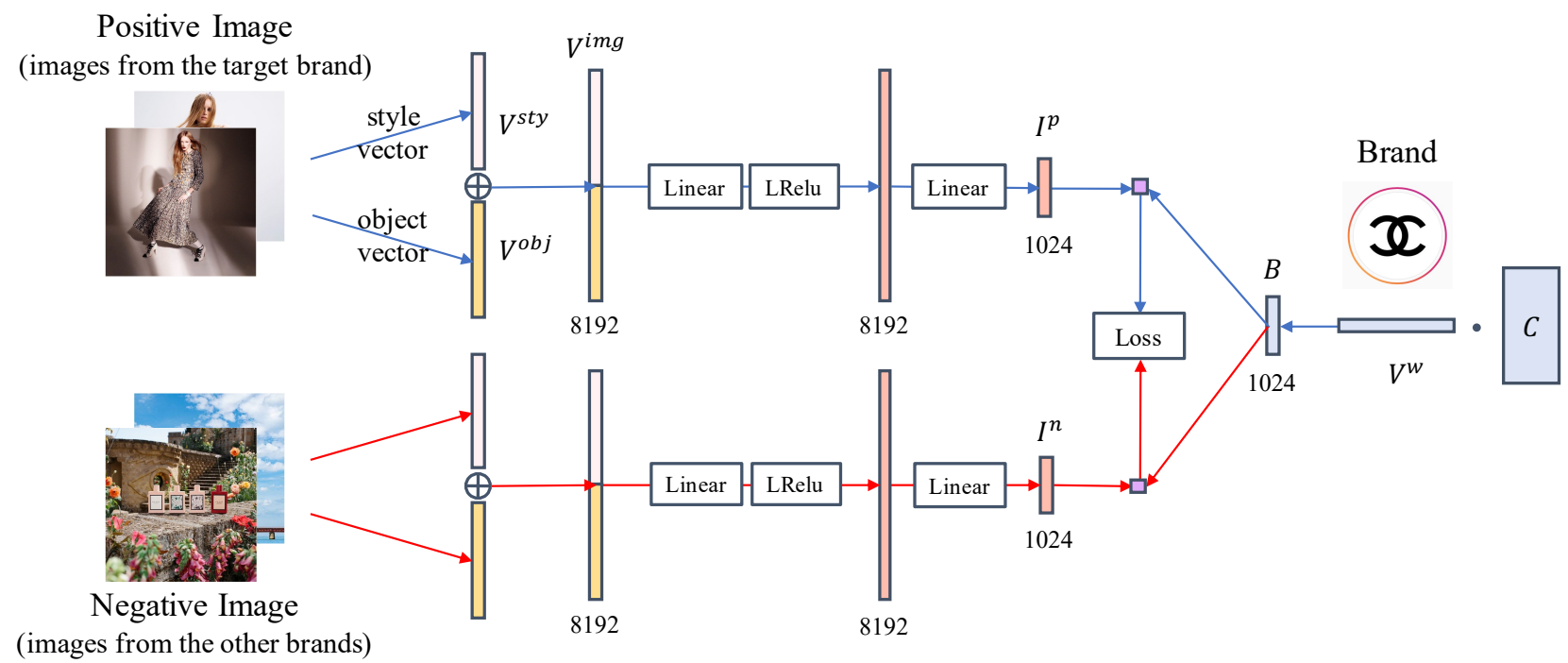

Figure 2: The proposed framework for the brand concept learning.

items [36]. Based on the BPR framework, visual BPR (VBPR) is proposed to enrich the item latent vectors with image features [17]. Several BPR-based models, such as DVBPR [19] and NPR [33], also exhibit good performance. Many works investigated image features in different recommendation applications. For example, Zhao et al. used image data in a movie recommendation task [44]. [1, 32] used product images in item recommendation, [3, 42] used image data in personalized fashion recommendation, and [41] used userinteracted images in social image recommendation. However, these methods are not purely content-based as they only integrate image data in traditional collaborative filtering-based frameworks.

Several works have performed image recommendation based solely on image content. Ding et al. used popular and unpopular image pairs to train a network that can recommend social images based on popularity [7]. Gelli et al. proposed a pairwise learning framework to recommend images to brands $[15,16]$. This work is similar to our scenario, but we found that their framework is based on image features extracted by pre-trained neural networks. We presume that such image features could accurately represent objects in the image but not the style of the image. Finding what kind of objects are posted by brands is not enough to understand the concept of a brand and recommend suitable images. In summary, image recommendation is of great value and appropriate methods are highly dependent on each application scenario. Therefore, further analysis of image style is needed for the brand image recommendation task.

\section{PROPOSED METHOD}

We propose a Style-Aware Image Recommendation (SAIR) framework as a novel image recommendation and discovery framework, which explicitly models the brand concept for social media marketing. The proposed framework is illustrated in Figure 2. The framework comprises two main parts: image feature extraction and representation learning. In the feature extraction part, we used both the object and style vectors to represent the image. In the representation learning part, the basic idea of the proposed method is similar to that of BPR [36]. The goal is to recommend images that match the brand concept, which is determined by the images posted by the brand in the past. Note that we only use brands in the notation for better understanding. When applied to influencers, only replacing the input is enough.

\subsection{Problem Formulation}

We denote $\mathcal{S}=\left\{b_{1}, b_{2}, \ldots, b_{|S|}\right\}$ the set of social media brand accounts, which consists of images $\mathcal{H}=\left\{i_{1}, i_{2}, \ldots, i_{|H|}\right\}$. And $\mathcal{H}_{b_{j}} \in \mathcal{H}$ indicates the images posted by brand $b_{j}$. Then, we generate a set of triplets $\left(b_{j}, i^{p}, i^{n}\right)$ to train the model, where $i^{p} \in \mathcal{H}_{b_{j}}$ and $i^{n} \notin \mathcal{H}_{b_{j}}$. That is, for a target brand and previously posted image (positive image), we randomly sample an image that is not posted by the target brand (negative image). For the target brand $b_{j}$, the learned brand representation is denoted by $\mathbf{B}_{j} . \mathbf{I}^{p}$ is the learned image representation for $i^{p}$, and $\mathbf{I}^{n}$ is the learned image representation for $i^{n}$.

The training of the model is based on the assumption that the learned brand representation $\mathbf{B}_{j}$ should be more similar to images posted by the target brand (the positive image representation $\mathrm{I}^{p}$ ) than images posted by the other brands (the negative image representation $\mathbf{I}^{n}$ ). If an image representation is similar to the brand representation, it is considered to match the brand concept. Therefore, the essence of our proposed brand image recommendation is to learn a ranking score function $f(b, i)$ as:

$$
f\left(b_{j}, i^{p}\right)>f\left(b_{j}, i^{n}\right) .
$$

\subsection{Image Feature Extraction}

3.2.1 Object Vector. We used the VGG16 network [38] pretrained on ImageNet [6] and extracted object features from the first FC 
feature maps (conv5_1)
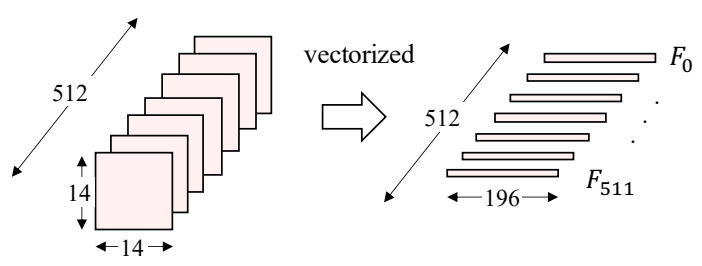

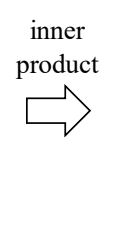

Gram matrix $G$

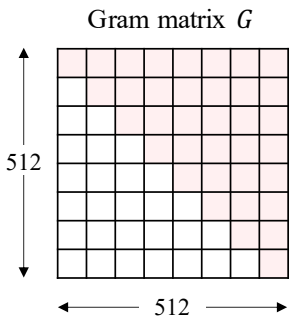

(symmetric matrix) vectorized upper triangular part

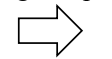

style vector $V^{\text {sty }}$
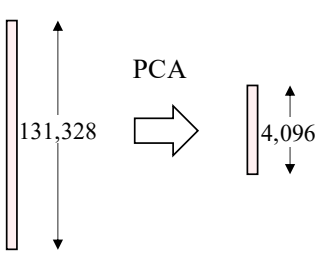

Figure 3: The process of style vector extraction.

layer. We transformed each image into a 4,096-dimension feature vector. The extracted object vector is denoted by $\mathrm{V}^{\text {obj }}$.

3.2.2 Style Vector. Similar to the object vector, we used the VGG16 network [38] pretrained on ImageNet [6] as the base network. Following previous work on neural style transfer [14], we implemented a feature space called the Gram matrix to capture the image texture information. Such features presumably represent brand styles more accurately than the final output of CNNs. Following [4], we transformed the exciting findings of the Gram matrix and used it as part of the image style feature extraction process.

The process of feature extraction of the style vector is shown in Figure 3. For example, the layer "conv5_1" of the VGG16 network consists of 512 feature maps. The width and height of each feature map are both 14, and the vectorized feature maps become $\mathrm{F} \in \mathcal{R}^{196}$. The Gram matrix is represented using $\mathrm{G} \in \mathcal{R}^{512 \times 512}$, where $\mathrm{G}_{j k}$ is the inner product of two vectorized feature maps $\mathbf{F}_{j}$ and $\mathbf{F}_{k}$. The Gram matrix is calculated as follows:

$$
\mathbf{G}_{j k}=\sum_{l} \mathbf{F}_{j l} \mathbf{F}_{k l},
$$

where $l$ is the index. The vectorized Gram matrix is a 262,144dimension vector; its dimension is too large for further training and needs to be reduced. Because the Gram matrix is symmetrical, we first extracted the upper triangular part and transformed it into a 131,328-dimension vector. Then, we applied incremental PCA [37], which is a memory-efficient PCA algorithm [39], and achieved reduction of the dimension to 4,096. Finally, the reduced vector was adopted as the style vector $\mathbf{V}^{\text {sty }}$.

Note that other layers such as "conv5_2" and "conv5_3", can also serve as input. Results obtained from different layers are shown and discussed in Section 5.3.

\subsection{Representation Learning}

3.3.1 Image Representation Learning. The object and style vectors of the image are denoted by $\mathbf{V}^{o b j}$ and $\mathbf{V}^{s t y}$, respectively. The representation of the image uses the concatenation of two vectors, and the image vector $\mathbf{V}^{i m g}$ can be calculated as follows:

$$
\mathbf{V}^{i m g}=\mathbf{V}^{o b j} \oplus \mathbf{V}^{s t y},
$$

where $\oplus$ is the concatenate function. Then, a two-layer neural network is used in image representation learning, and the image representation $\mathbf{I} \in \mathcal{R}^{1024}$ is calculated as follows:

$$
\mathbf{I}=\mathbf{W}_{2}\left(\phi\left(\mathbf{W}_{1} \mathbf{V}^{i m g}+\mathbf{y}_{1}\right)\right)+\mathbf{y}_{2},
$$

where $\phi$ denotes a nonlinear activation function, $\mathbf{W}_{1} \in \mathcal{R}^{8912 \times 8912}$, $\mathbf{y}_{1} \in \mathcal{R}^{8912}, \mathbf{W}_{2} \in \mathcal{R}^{1024 \times 8912}, \mathbf{y}_{2} \in \mathcal{R}^{1024}$. Both the positive image representation $\mathrm{I}^{p}$ and the negative image representation $\mathrm{I}^{n}$ utilize the same network.

3.3.2 Brand Representation Learning. Unlike image representation learning, brand representation learning does not include feature extraction. Some recommendation approaches learn user latent vectors directly from a one-hot ID matrix [17, 33], but [16] demonstrated that such an approach fails to learn brand representation in the same category, they instead used both the one-hot brand ID and an association matrix to learn brand representation. Therefore, we used a similar method in order to capture the brand concept. We define $\mathbf{V}_{j}^{w} \in \mathcal{R}^{2000}$ as the weight vector of brand $b_{j}$, and the concept matrix $C \in \mathcal{R}^{2000 \times 1024}$ for the brand representation learning. For a target brand $b_{j}$, the final brand representation $\mathbf{B}_{j}$ is computed as:

$$
\mathbf{B}_{j}=\mathbf{V}_{j}^{w} \mathbf{C} \text {. }
$$

3.3.3 Optimization. The similarity score between image $i \in \mathcal{H}$ and any brand $b \in \mathcal{S}$ is calculated using the cosine similarity and computed as follows:

$$
f(b, i)=\frac{\mathbf{B}^{\top} \mathbf{I}}{\|\mathbf{B}\| \cdot\|\mathbf{I}\|} .
$$

For a target brand $b_{j}$, the learned representations of the triplets $\left(b_{j}, i^{p}, i^{n}\right)$ are denoted by $\left(\mathbf{B}_{j}, \mathbf{I}^{p}, \mathbf{I}^{n}\right)$. A pairwise learning framework was used to train our model; i.e., the positive image was expected to score higher than the negative image. The model was trained using the margin ranking loss, as follows:

$$
\mathcal{L}=\max \left(0, f\left(b_{j}, i^{n}\right)-f\left(b_{j}, i^{p}\right)+m\right)+\lambda\left\|\mathbf{V}^{w}\right\|_{1},
$$

where $m$ is the minimum margin desired between positive and negative images. In addition, we adopted an L1 regularization on $\mathbf{V}^{w}$ to encourage sparse representation.

3.3.4 Implementation Details. For a target brand and positive image pair, we randomly sampled ten negative images for training. Uniform negative sampling was adopted. The model was trained for twenty epochs and used a batch size of 256. We used Adam [23] as the optimizer with a learning rate of $10^{-5}$ and decays by 0.5 every 5 epochs, weight decay was set to $10^{-4}$. The margin $m$ was set to 0.3 , and leaky ReLU was adopted as the activation function $\phi$. A dropout layer with a rate of 0.5 was used, and the L1 regularization rate $\lambda$ was set to $10^{-4}$. 
Table 1: Summary of Instagram datasets.

\begin{tabular}{ccccc}
\hline Dataset & \#brands & \#categories & \#train images & \#test images \\
\hline Brand & 813 & 14 & $1,206,760$ & 8,130 \\
Influencer & 616 & 8 & 933,055 & 6,160 \\
\hline
\end{tabular}

\section{DATASET CONSTRUCTION}

Instagram is one of the most popular social media platforms for brand marketing. Therefore, we conducted our experiments using two large-scale Instagram datasets. The details of two Instagram datasets are summarized in Table 1.

For the brand dataset, we started with the Instagram brand dataset from the work [16]. Because only the brand name and image IDs were released, images and publicly available data were crawled through the official API of Instagram. Using the brand list, we crawled the latest 2,000 images of each brand account. We removed brands with less than 100 images. The new crawled dataset consists of 813 brands and 1,214,890 images.

In order to prove that the proposed framework could be applied to other tasks such as influencer marketing by only changing the input, we collected another large-scale Instagram dataset in this paper. We started with a Instagram influencer dataset from the work [22], they provided an influencer list consisting of 33,935 influencers. Many influencers in their list only have hundreds of followers, and we think these influencers are not representative. Hence, we removed influencers with less than 100 images and 500,000 followers. The final influencer list consists of 616 influencers in eight categories. Using the influencer list, we crawled the latest 2,000 images of each influencer account. The crawled dataset consists of 616 influencers and 939,215 images.

\section{EVALUATION}

\subsection{Evaluation Protocols}

We split the dataset into training and testing sets; the test set contained the 10 most recent images of each brand/influencer, while the remaining data were used for training. This resulted in 1,206,760 training images and 8,130 testing images for the brand dataset, 933,055 training images and 6,160 testing images for the influencer dataset.

Take the brand dataset as an example. The test images are denoted by $\mathcal{H}^{\text {test }}=\left\{i_{1}, i_{2}, \ldots, i_{8130}\right\}$, and $\mathcal{H}_{b}^{\text {test }} \in \mathcal{H}^{\text {test }}$ indicates the images are posted by the brand $b$. The experimental evaluation mainly focuses on ranking all test images for each brand $b \in \mathcal{S}$.

We introduced AUC, cAUC, HR@k, NDCG@k, and MedR to evaluate the recommendation performance.

- AUC: represents the probability that a positive image will rank higher than a negative image.

$$
A U C=\frac{1}{|S|} \sum_{b} \frac{1}{|E(b)|} \sum_{\left(i^{p}, i^{n}\right) \in E(b)} \delta\left(f\left(b, i^{p}\right)>f\left(b, i^{n}\right)\right),
$$

where $\delta$ is the indicator function, and the evaluation pairs per brand $b$ are:

$$
E(b)=\left\{\left(i^{p}, i^{n}\right) \mid i^{p} \in \mathcal{H}_{b}^{\text {test }} \wedge i^{n} \in \mathcal{H}_{c}^{\text {test }}, c \neq b\right\} .
$$

- cAUC: is the AUC that restricts the evaluation pairs to brands belonging to the same category. cAUC is computed in a similar way to AUC, with the exception of the following part:

$$
E(b)=\left\{\left(i^{p}, i^{n}\right) \mid i^{p} \in \mathcal{H}_{b}^{\text {test }} \wedge i^{n} \in \mathcal{H}_{c}^{\text {test }}, V(c)=V(b)\right\},
$$

where $V(b)$ is the category of the brand $b$.

- HR@k: represents the hit ratio, which is the fraction of positive images that have been recommended within the top $k$ images. $\mathcal{H}_{b}^{\text {topk }} \in \mathcal{H}_{b}^{\text {test }}$ indicates that a set of images posted by brand $b$ are ranked in the top $k$ recommendation list to the brand.

$$
H R @ k=\frac{1}{|S|} \sum_{b} \frac{\left|\mathcal{H}_{b}^{t o p k}\right|}{\left|\mathcal{H}_{b}^{\text {test }}\right|},
$$

- NDCG@k: represents the normalized discounted cumulative gain [18]. It is used to evaluate the performance of image recommendation by accounting for the ranking of relevant images.

- MedR: is the median position of the first positive image. The lower the MedR is, the better the model performs.

\subsection{Comparison to Previous Work}

To demonstrate the effectiveness of the proposed method, we adopted the following methods as baselines.

- Random: we generated a random ranking for test images.

- AvgVec-obj: we averaged image object vectors $\left(\mathrm{V}^{o b j}\right)$ as the brand vector. Then, we computed the cosine similarity score between the brand vector and test image object vector to create a recommendation list.

- AvgVec-sty: we averaged image style vectors $\left(\mathbf{V}^{\text {sty }}\right)$ as the brand vector. Then, we computed the cosine similarity score between the brand vector and test image style vector to create a recommendation list.

- PCD [16]: this is a state-of-the-art method for brand image recommendation. The image feature extracted from the pretrained VGG16 network was used as input, and the brand representation was trained on a pairwise learning network. We used the implementation released by the authors.

- PCD-opt [16]: this is a variant of PCD [16]. In the original paper and implementation, they used Adadelta as the optimizer, but we found that using Adam provides better results in the experiments. Therefore, we designed a variant of their framework that use the same optimizer setting as in our proposed method for a fair comparison.

- SAIR: this is our proposed method.

The comparative results presented in Table 2 indicate that the proposed method, which combines object and style representation, yields the best performance in both datasets. It is also observed that the cAUCs are consistently lower than the AUCs. This is mainly because cAUC focuses on evaluating the ability of differentiating images within the same brand/influencer category, which is a tougher task than image recommendation in all categories.

We observe that PCD-opt [16] exhibited a good AUC but low HR and NDCG, implying that their method can rank test images relatively well but cannot achieve high performance, especially for 
Table 2: Comparison of the proposed method with the baselines.

\begin{tabular}{|c|c|c|c|c|c|c|c|c|c|c|}
\hline & \multicolumn{5}{|c|}{ Brand Dataset } & \multicolumn{5}{|c|}{ Influencer Dataset } \\
\hline & AUC & cAUC & HR@10 & NDCG@10 & MedR & AUC & cAUC & HR@10 & NDCG@10 & MedR \\
\hline Random & 0.497 & 0.497 & 0.001 & 0.001 & 529 & 0.501 & 0.501 & 0.002 & 0.003 & 394 \\
\hline AvgVec-obj & 0.786 & 0.680 & 0.065 & 0.076 & 26 & 0.648 & 0.609 & 0.021 & 0.025 & 139.5 \\
\hline AvgVec-sty & 0.808 & 0.700 & 0.072 & 0.088 & 21 & 0.732 & 0.682 & 0.040 & 0.049 & 62.5 \\
\hline $\mathrm{PCD}[16]$ & 0.885 & 0.747 & 0.076 & 0.081 & 16 & 0.837 & 0.785 & 0.063 & 0.068 & 22 \\
\hline PCD-opt [16] & 0.918 & 0.795 & 0.123 & 0.143 & 7 & 0.865 & 0.818 & 0.088 & 0.100 & 12 \\
\hline SAIR & 0.946 & 0.856 & 0.299 & 0.353 & 1 & 0.918 & 0.885 & 0.279 & 0.350 & 1 \\
\hline
\end{tabular}

Table 3: Results of variants of the proposed method.

\begin{tabular}{|c|c|c|c|c|c|c|c|c|c|c|}
\hline & \multicolumn{5}{|c|}{ Brand Dataset } & \multicolumn{5}{|c|}{ Influencer Dataset } \\
\hline & AUC & cAUC & HR@10 & NDCG@10 & MedR & AUC & cAUC & HR@10 & NDCG@10 & MedR \\
\hline SAIR-obj & 0.928 & 0.810 & 0.161 & 0.190 & 4 & 0.866 & 0.817 & 0.110 & 0.129 & 11 \\
\hline SAIR-sty (conv5_1) & 0.938 & 0.822 & 0.202 & 0.239 & 3 & 0.912 & 0.872 & 0.185 & 0.224 & 3 \\
\hline SAIR-sty (conv5_2) & 0.937 & 0.820 & 0.193 & 0.229 & 3 & 0.903 & 0.861 & 0.168 & 0.203 & 3 \\
\hline SAIR-sty (conv5_3) & 0.932 & 0.813 & 0.191 & 0.223 & 3 & 0.892 & 0.848 & 0.151 & 0.177 & 5 \\
\hline SAIR-plus & 0.942 & 0.842 & 0.256 & 0.302 & 2 & 0.917 & 0.883 & 0.248 & 0.306 & 1 \\
\hline SAIR-concat & 0.946 & 0.856 & 0.299 & 0.353 & 1 & 0.918 & 0.885 & 0.279 & 0.350 & 1 \\
\hline
\end{tabular}

all test images shown in the top 10 recommendation list. In both datesets, our proposed method is about three times larger than PCD-opt [16] in terms of HR@10.

We found that the results in the brand dataset are generally better than the results in the influencer dataset. We also found that both AvgVec-obj and AvgVec-sty work extremely bad in the influencer dataset. We think this might be because some individual influencers do not have professional groups to help them create images, hence naive methods are hard to capture their concept.

Finally, the proposed method has the best HR and NDCG values and the lowest MedR, indicating that the learned brand/influencer and image representation have a higher capability of recommending images that match the brand/influencer concept.

\subsection{Variants of Proposed Method}

In this section, we compare the performance of the object and style vectors of different layers. In the proposed method, we concatenated the object and style vectors (conv5_1) as the image vector. Here, we only used one feature as input to show the individual performance of each vector. For example, when using object vector $\mathrm{V}^{o b j}$ as the input, the image representation $I$ is calculated as follows:

$$
\mathbf{I}=\hat{\mathbf{W}}_{2}\left(\phi\left(\hat{\mathbf{W}}_{1} \mathbf{V}^{o b j}+\hat{\mathbf{y}}_{1}\right)\right)+\hat{\mathbf{y}}_{2},
$$

where $\hat{\mathbf{W}}_{1} \in \mathcal{R}^{4096 \times 4096}, \hat{\mathbf{y}}_{1} \in \mathcal{R}^{4096}, \hat{\mathbf{W}}_{2} \in \mathcal{R}^{1024 \times 4096}, \hat{\mathbf{y}}_{2} \in \mathcal{R}^{1024}$, and the other part is the same as our proposed method. In addition, we show the results of the style vector from the layers "conv5_2" and "conv5_3".

We also conducted an experiment to compare using different combination of the object and style vectors as the input of SAIR. Besides using concatenation in the proposed method (SAIR-concat) as described in previous section, we use element-wise addition of the object and style vectors in this experiment (SAIR-plus).

The results presented in Table 3 indicate that using style vectors (SAIR-sty) generally perform better than object vectors (SAIR-obj) in both datasets. This finding proves our assumption that the image style vector can represent a brand/influencer concept more accurately than the object vector. In the influencer dataset, we found that style vectors perform much better than object vectors. We think it's because one influencer might cooperate with several brands, and various objects might appear in the images. On the other hand, brands only post their own products, hence object vectors are more effective in brand dataset than in influencer dataset. In both datasets, the style vector derived from "conv5_1" performs the best; therefore, it was used in the proposed method.

When using different combinations of the object and style vectors, we found that concatenation (SAIR-concat) showed better results than element-wise addition (SAIR-plus).

\section{CASE STUDIES}

\subsection{Performance in Different Categories}

Table 4 shows the performance of the proposed method for brands in different categories. We found that our framework performs the best in terms of image recommendation for the furniture category. And our framework performs not so well in the finance category. The auto category exhibits a high AUC but low cAUC, suggesting that images are rather difficult to differentiate within this category but easily distinguished from images of the other categories. The same applies to the airlines category. The fashion category exhibited a high AUC but low HR, implying that test images ranking is 
Table 4: Performance in different brand categories.

\begin{tabular}{cccccc}
\hline Category & AUC & cAUC & HR@10 & NDCG@10 & MedR \\
\hline fashion & 0.954 & 0.889 & 0.256 & 0.309 & 2 \\
alcohol & 0.954 & 0.817 & 0.381 & 0.474 & 1 \\
entertainment & 0.958 & 0.914 & 0.347 & 0.405 & 1 \\
food & 0.941 & 0.858 & 0.395 & 0.472 & 1 \\
auto & 0.965 & 0.830 & 0.291 & 0.330 & 2 \\
energy & 0.920 & 0.920 & 0.267 & 0.284 & 10 \\
beverages & 0.945 & 0.896 & 0.346 & 0.422 & 1 \\
airlines & 0.936 & 0.678 & 0.149 & 0.173 & 7 \\
jewelry & 0.966 & 0.879 & 0.327 & 0.380 & 1 \\
furniture & 0.976 & 0.903 & 0.450 & 0.504 & 1 \\
services & 0.923 & 0.898 & 0.264 & 0.312 & 2 \\
electronics & 0.914 & 0.825 & 0.214 & 0.248 & 3 \\
nonprofit & 0.950 & 0.883 & 0.307 & 0.377 & 1 \\
finance & 0.898 & 0.760 & 0.161 & 0.203 & 6 \\
\hline
\end{tabular}

Table 5: Performance in different influencer categories.

\begin{tabular}{cccccc}
\hline Category & AUC & cAUC & HR@10 & NDCG@10 & MedR \\
\hline beauty & 0.947 & 0.896 & 0.450 & 0.549 & 1 \\
family & 0.916 & 0.852 & 0.244 & 0.328 & 1 \\
fashion & 0.905 & 0.895 & 0.242 & 0.308 & 1 \\
fitness & 0.944 & 0.908 & 0.414 & 0.507 & 1 \\
food & 0.969 & 0.846 & 0.347 & 0.425 & 1 \\
interior & 0.975 & 0.839 & 0.318 & 0.395 & 1 \\
pet & 0.985 & 0.913 & 0.788 & 0.811 & 1 \\
travel & 0.943 & 0.856 & 0.277 & 0.321 & 2 \\
\hline
\end{tabular}

achieved relatively well. However, achieving high performance is hard, especially for all test images shown in the top 10 recommendation list.

Table 5 shows the performance of the proposed method for influencers in different categories. We found that our framework performs extremely well in the pet category, this might be because influencers in this category have a unique personality comparing to influencers in the other categories. Because both sponsored and non-sponsored posts are focusing on the influencer (pet), their concept consistency is maintained very well.

\subsection{Recommendation Examples}

In this section, we present examples of the top 10 images recommended to brands and influencers by the proposed method. This setting actually shows the results of HR@10. Note that the test dataset consists of 10 previously posted images per brand/influencer. Thus, the most successful case is that all 10 test images appear in the top 10 recommendation list. We show several successful and unsuccessful examples in Figure 4. The Instagram account name is shown above each image, and the ground truth brand/influencer are marked in red.

Figures 4(a), (b), (c), and (d) are recommendation results in the brand dataset. Figure 4(a) presents the top 10 images recommended to the brand Panda Silk. We can see that nine images have actually been posted by Panda Silk. As discussed in Section 6.1, most brands in the furniture category have a unique style that is much easier to recognize. Figures 4(b) and (c) present the top 10 images recommended to the brand SHAKE SHACK and BURGER KING, respectively. Both brands posted images related to hamburgers, but we can see that they have different styles. SHAKE SHACK prefers light color backgrounds while BURGER KING prefers dark color backgrounds. For SHAKE SHACK, six of the test images are included in the top 10 list, indicating relatively good performance. We present a recommendation example that underperformed in Figure 4(d), which is the result of a fashion brand Chanel. Because the brand often posts black and white images, some negative images in black and white were recommended. According to the results, images in black and white are very similar and hard to distinguish from each other.

Figures 4(e), (f), and (g) are recommendation results in the influencer dataset. Figure 4(e) presents the top 10 images recommended to the pet influencer Doug The Pug. We can see that eight images have actually been posted by Doug The Pug. As discussed in Section 6.1, recommendation results are generally good in the pet category because of pet influencers' unique style. Figure 4(f) presents the top 10 images recommended to the beauty influencer Denitslava. This influencer also has a very unique style, which assists appropriate image recommendation. We present a recommendation example that underperformed in Figure 4(g), which is the result of a travel influencer Andy Best. We found that influencers in the travel category tend to only post landscape images and most of them are very similar, which allowed some negative images to rank in the top list.

\section{CONCLUSIONS AND FUTURE WORK}

In this paper, we proposed a framework called Style-Aware Image Recommendation (SAIR) to recommend images that match the concept of brands or influencers for effective social media marketing. We investigated the usefulness of style features for image recommendation and discovery. The proposed method can learn brands or influencers representation from the images posted by them on social media, and help them automatically choosing image candidates matching their concept to post or repost. Experiments on two large-scale Instagram datasets demonstrated that the proposed method SAIR outperforms the state-of-the-art methods and succeeds in recommending images that fit the concept of the target brand or influencer.

In future work, we intend to incorporate temporal information in the proposed method because brand/influencer concepts might change over time. Second, we intend to add popularity information because we believe that recommend high-popularity images are an attractive topic.

\section{ACKNOWLEDGMENTS}

This work was partially supported by the Grants-in-Aid for Scientific Research Numbers JP19J22939 and JP18H03339. 


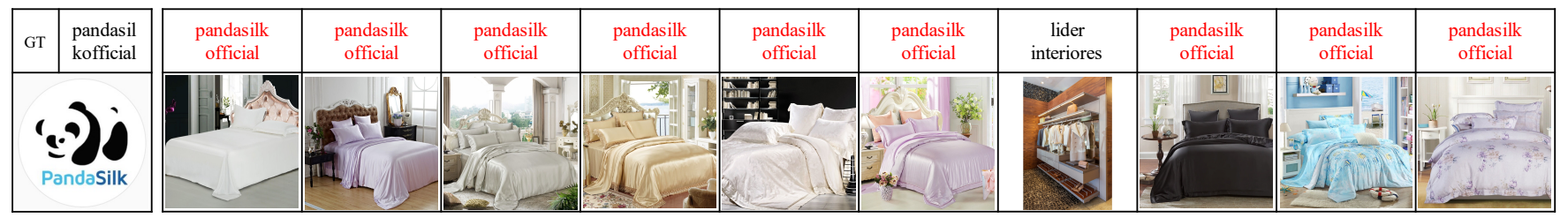

(a) Top 10 images recommended to the brand Panda Silk.

\begin{tabular}{|l|l|l|l|l|l|l|l|l|l|}
\hline GT & $\begin{array}{l}\text { shake } \\
\text { shack }\end{array}$ \\
\hline shakeshack & shakeshack & shakeshack & shakeshack & shakeshack & zagat & shakeshack & $\begin{array}{c}\text { rotary } \\
\text { international }\end{array}$ & burgerking & pringlesus \\
\hline
\end{tabular}

(b) Top 10 images recommended to the brand SHAKE SHACK.

\begin{tabular}{|l|l|l|l|l|l|l|l|l|l|}
\hline GT & $\begin{array}{c}\text { burger } \\
\text { king }\end{array}$ \\
\hline Nff_canada & burgerking & $\begin{array}{c}\text { mcdonald } \\
\text { ssverige }\end{array}$ & medo_ph & burgerking & $\begin{array}{c}\text { modonalds } \\
\text { austria }\end{array}$ & burgerking & ihop & arbys & burgerking \\
\hline
\end{tabular}

(c) Top 10 images recommended to the brand BURGER KING.

\begin{tabular}{|c|c|c|c|c|c|c|c|c|c|}
\hline GT $\begin{array}{c}\text { chanel } \\
\text { official }\end{array}$ & graff & $\begin{array}{c}\text { chanel } \\
\text { official }\end{array}$ & dior & $\begin{array}{c}\text { thesociety } \\
\text { nyc }\end{array}$ & dior & $\begin{array}{c}\text { aliciahannah } \\
\text { naomi }\end{array}$ & $\begin{array}{c}\text { maison } \\
\text { tabbah }\end{array}$ & $\begin{array}{c}\text { bobbyarthur } \\
\text { jewelry }\end{array}$ & $\begin{array}{c}\text { giorgio } \\
\text { armani }\end{array}$ \\
\hline
\end{tabular}

(d) Top 10 images recommended to the brand Chanel.

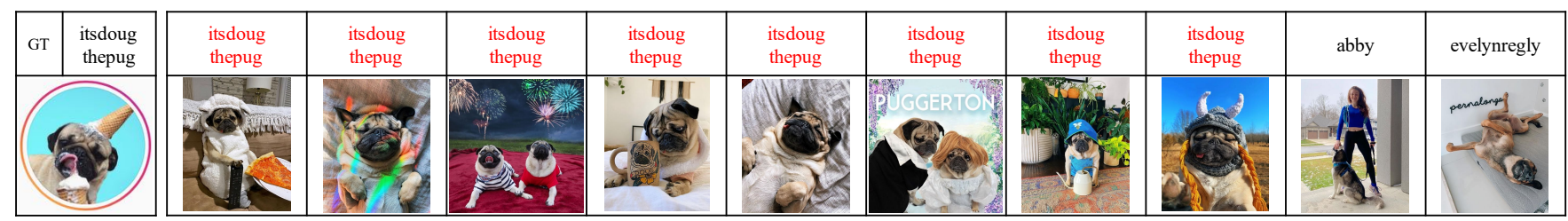

(e) Top 10 images recommended to the influencer Doug The Pug.

\begin{tabular}{|l|l|l|l|l|l|l|l|l|l|}
\hline GT & $\begin{array}{c}\text { denits } \\
\text { lava }\end{array}$ \\
\hline & denitslava & denitlava & denitslava & denitslava & denitslava & denitslava & $\begin{array}{c}\text { cosmoby } \\
\text { haley }\end{array}$ & $\begin{array}{c}\text { valebigella } \\
\text { peachyqueen } \\
\text { blog }\end{array}$ & \begin{tabular}{c} 
denitslava \\
\hline
\end{tabular} \\
\hline
\end{tabular}

(f) Top 10 images recommended to the influencer Denitslava.

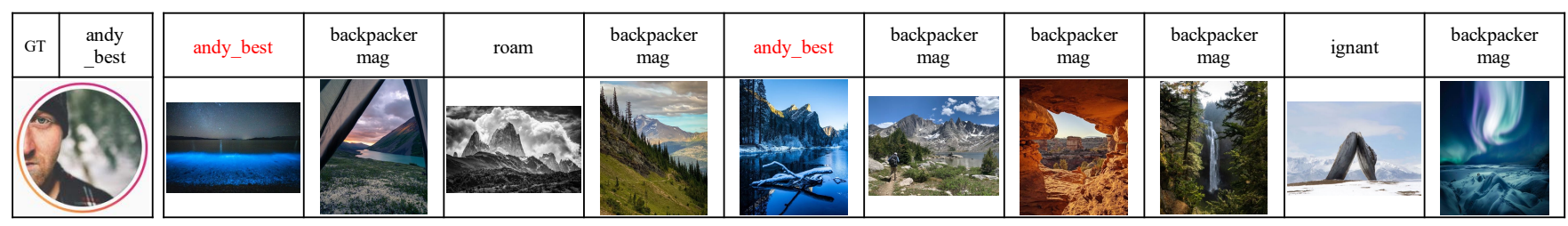

(g) Top 10 images recommended to the influencer Andy Best.

Figure 4: Examples of top 10 recommended images to brands and influencers. 


\section{REFERENCES}

[1] Jingyuan Chen, Hanwang Zhang, Xiangnan He, Liqiang Nie, Wei Liu, and TatSeng Chua. 2017. Attentive collaborative filtering: Multimedia recommendation with item-and component-level attention. In Proceedings of the International ACM SIGIR Conference on Research and Development in Information Retrieval (SIGIR). 335-344.

[2] Liyi Chen and Jufeng Yang. 2019. Recognizing the Style of Visual Arts via Adaptive Cross-layer Correlation. In Proceedings of the ACM International Conference on Multimedia (ACMMM). 2459-2467.

[3] Xu Chen, Hanxiong Chen, Hongteng Xu, Yongfeng Zhang, Yixin Cao, Zheng Qin, and Hongyuan Zha. 2019. Personalized fashion recommendation with visual explanations based on multimodal attention network: Towards visually explainable recommendation. In Proceedings of the International ACM SIGIR Conference on Research and Development in Information Retrieval (SIGIR). 765774.

[4] Wei-Ta Chu and Yi-Ling Wu. 2016. Deep correlation features for image style classification. In Proceedings of the ACM International Conference on Multimedia (ACMMM). 402-406.

[5] Lisette De Vries, Sonja Gensler, and Peter SH Leeflang. 2012. Popularity of brand posts on brand fan pages: An investigation of the effects of social media marketing. Fournal of Interactive Marketing 26, 2 (2012), 83-91.

[6] Jia Deng, Wei Dong, Richard Socher, Li-Jia Li, Kai Li, and Li Fei-Fei. 2009. Imagenet: A large-scale hierarchical image database. In Proceedings of the IEEE Conference on Computer Vision and Pattern Recognition (CVPR). 248-255.

[7] Keyan Ding, Kede Ma, and Shiqi Wang. 2019. Intrinsic Image Popularity Assessment. In Proceedings of the ACM International Conference on Multimedia (ACMMM). 1979-1987.

[8] Yi Ding, Chee Wei Phang, Xianghua Lu, Chuan-Hoo Tan, and Juliana Sutanto. 2014. The role of marketer-and user-generated content in sustaining the growth of a social media brand community. In Proceedings of the Hawaii International Conference on System Sciences. 1785-1792.

[9] Aleksandr Farseev, Kirill Lepikhin, Hendrik Schwartz, Eu Khoon Ang, and Kenny Powar. 2018. SoMin. ai: Social Multimedia Influencer Discovery Marketplace. In Proceedings of the ACM International Conference on Multimedia (ACMMM) 1234-1236.

[10] Reto Felix, Philipp A Rauschnabel, and Chris Hinsch. 2017. Elements of strategic social media marketing: A holistic framework. Journal of Business Research 70 (2017), 118-126

[11] Tian Gan, Shaokun Wang, Meng Liu, Xuemeng Song, Yiyang Yao, and Liqiang Nie. 2019. Seeking Micro-influencers for Brand Promotion. In Proceedings of the ACM International Conference on Multimedia (ACMMM). 1933-1941.

[12] Leon Gatys, Alexander S Ecker, and Matthias Bethge. 2015. Texture synthesis using convolutional neural networks. In Advances in neural information processing systems (NIPS). 262-270.

[13] Leon A Gatys, Alexander S Ecker, and Matthias Bethge. 2015. A neural algorithm of artistic style. arXiv preprint arXiv:1508.06576 (2015).

[14] Leon A Gatys, Alexander S Ecker, and Matthias Bethge. 2016. Image style transfer using convolutional neural networks. In Proceedings of the IEEE Conference on Computer Vision and Pattern Recognition (CVPR). 2414-2423.

[15] Francesco Gelli, Tiberio Uricchio, Xiangnan He, Alberto Del Bimbo, and Tat-Seng Chua. 2020. Learning visual elements of images for discovery of brand posts. ACM Transactions on Multimedia Computing, Communications, and Applications (TOMM) 16, 2 (2020), 1-21.

[16] Francesco Gelli, Tiberio Uricchio, Xiangnan He, Alberto Del Bimbo, and TatSeng Chua. 2018. Beyond the product: discovering image posts for brands in social media. In Proceedings of the ACM International Conference on Multimedia (ACMMM). 465-473.

[17] Ruining He and Julian McAuley. 2016. VBPR: visual Bayesian Personalized Ranking from implicit feedback. In Proceedings of the AAAI Conference on Artificial Intelligence (AAAI). 144-150.

[18] Kalervo Järvelin and Jaana Kekäläinen. 2002. Cumulated gain-based evaluation of IR techniques. ACM Transactions on Information Systems (TOIS) 20, 4 (2002), 422-446.

[19] Wang-Cheng Kang, Chen Fang, Zhaowen Wang, and Julian McAuley. 2017. Visually-aware fashion recommendation and design with generative image models. In IEEE International Conference on Data Mining (ICDM). 207-216.

[20] Sergey Karayev, Matthew Trentacoste, Helen Han, Aseem Agarwala, Trevor Darrell, Aaron Hertzmann, and Holger Winnemoeller. 2013. Recognizing image style. arXiv preprint arXiv:1311.3715 (2013).
[21] Gunhee Kim and Eric P Xing. 2014. Visualizing brand associations from web community photos. In Proceedings of the ACM international conference on Web search and data mining. 623-632.

[22] Seungbae Kim, Jyun-Yu Jiang, Masaki Nakada, Jinyoung Han, and Wei Wang. 2020. Multimodal Post Attentive Profiling for Influencer Marketing. In Proceedings of the International Conference on World Wide Web (WWW). 2878-2884.

[23] Diederik P Kingma and Jimmy Ba. 2014. Adam: A method for stochastic optimization. arXiv preprint arXiv:1412.6980 (2014).

[24] Even Johan Lanseng and Lars Erling Olsen. 2012. Brand alliances: the role of brand concept consistency. European fournal of Marketing (2012).

[25] Michel Laroche, Mohammad Reza Habibi, and Marie-Odile Richard. 2013. To be or not to be in social media: How brand loyalty is affected by social media? International fournal of Information Management 33, 1 (2013), 76-82.

[26] Michel Laroche, Mohammad Reza Habibi, Marie-Odile Richard, and Ramesh Sankaranarayanan. 2012. The effects of social media based brand communities on brand community markers, value creation practices, brand trust and brand loyalty. Computers in Human Behavior 28, 5 (2012), 1755-1767.

[27] Jingyuan Li, Ning Wang, Lefei Zhang, Bo Du, and Dacheng Tao. 2020. Recurrent feature reasoning for image inpainting. In Proceedings of the IEEE Conference on Computer Vision and Pattern Recognition (CVPR). 7760-7768.

[28] Yan Liu, Krista J Li, Haipeng Chen, and Subramanian Balachander. 2017. The effects of products' aesthetic design on demand and marketing-mix effectiveness: The role of segment prototypicality and brand consistency. Fournal of Marketing 81, 1 (2017), 83-102.

[29] David G Lowe. 2004. Distinctive image features from scale-invariant keypoints. International fournal of Computer Vision (IFCV) 60, 2 (2004), 91-110.

[30] Insa Matthiesen and Ian Phau. 2005. The 'HUGO BOSS'connection: Achieving global brand consistency across countries. Fournal of Brand Management 12, 5 (2005), 325-338.

[31] Masoud Mazloom, Robert Rietveld, Stevan Rudinac, Marcel Worring, and Willemijn Van Dolen. 2016. Multimodal popularity prediction of brand-related social media posts. In Proceedings of the ACM International Conference on Multimedia (ACMMM). 197-201.

[32] Julian McAuley, Christopher Targett, Qinfeng Shi, and Anton Van Den Hengel. 2015. Image-based recommendations on styles and substitutes. In Proceedings of the International ACM SIGIR Conference on Research and Development in Information Retrieval (SIGIR). 43-52.

[33] Wei Niu, James Caverlee, and Haokai Lu. 2018. Neural personalized ranking for image recommendation. In Proceedings of the ACM International Conference on Web Search and Data Mining. 423-431.

[34] Aude Oliva and Antonio Torralba. 2001. Modeling the shape of the scene: A holistic representation of the spatial envelope. International fournal of Computer Vision (IFCV) 42, 3 (2001), 145-175.

[35] Frank Palermo, James Hays, and Alexei A Efros. 2012. Dating historical color images. In European Conference on Computer Vision. Springer, 499-512.

[36] Steffen Rendle, Christoph Freudenthaler, Zeno Gantner, and Lars Schmidt-Thieme. 2009. BPR: Bayesian personalized ranking from implicit feedback. In Proceedings of the Conference on Uncertainty in Artificial Intelligence (UAI). 452-461.

[37] David A Ross, Jongwoo Lim, Ruei-Sung Lin, and Ming-Hsuan Yang. 2008. Incremental learning for robust visual tracking. International fournal of Computer Vision (IJCV) 77, 1-3 (2008), 125-141.

[38] Karen Simonyan and Andrew Zisserman. 2014. Very deep convolutional networks for large-scale image recognition. arXiv preprint arXiv:1409.1556 (2014).

[39] Michael E Tipping and Christopher M Bishop. 1999. Probabilistic principal component analysis. Journal of the Royal Statistical Society: Series B (Statistical Methodology) 61, 3 (1999), 611-622.

[40] Steven Woods. 2016. \# Sponsored: The emergence of influencer marketing. (2016).

[41] Le Wu, Lei Chen, Richang Hong, Yanjie Fu, Xing Xie, and Meng Wang. 2019. A hierarchical attention model for social contextual image recommendation. IEEE Transactions on Knowledge and Data Engineering (TKDE) (2019).

[42] Wenhui Yu, Huidi Zhang, Xiangnan He, Xu Chen, Li Xiong, and Zheng Qin. 2018. Aesthetic-based clothing recommendation. In Proceedings of the International Conference on World Wide Web (WWW). 649-658.

[43] Yiwei Zhang, Xueting Wang, Yoshiaki Sakai, and Toshihiko Yamasaki. 2019. Measuring Similarity between Brands using Followers' Post in Social Media. In Proceedings of the ACM International Conference on Multimedia Asia (ACMMMAsia). 1-6.

[44] Lili Zhao, Zhongqi Lu, Sinno Jialin Pan, and Qiang Yang. 2016. Matrix factorization+ for movie recommendation. In Proceedings of the International foint Conference on Artifical Intelligence (IfCAI). 3945-3951. 